\title{
Visibilidad e impacto altmétrico de los investigadores de la Universidad de Antioquia: metodología aplicable a universidades
}

\author{
Visibility and altmetric impact of the University of \\ Antioquia researchers: Methodology \\ applicable to universities
}

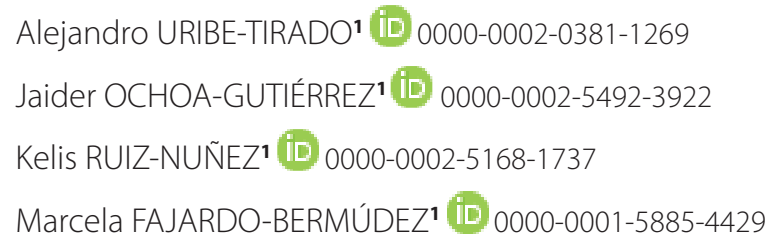

\section{Resumen}

Este trabajo es resultado de uno de los componentes de una investigación macro que busca crear un Modelo para identificar el grado de vinculación de una universidad con su entorno, en este caso, aplicado a la Universidad de Antioquia (Medellín-Colombia). Este componente se refiere a la visibilidad e impacto de los investigadores desde la perspectiva y datos que ofrecen las altmetrics. Para ello, se analizan los datos altmétricos de 1.032 investigadores de seis áreas del conocimiento, considerando diferentes plataformas académicas, profesionales y sociales, además de una plataforma integradora de indicadores altmétricos. Aunque se realiza esta medición para investigadores de la Universidad de Antioquia, la metodología general para la captura e interpretación de datos puede aplicarse a otras universidades que comparten características de investigación y de comunicación científica y que, a partir de las altmetrics, desean identificar la visibilidad e impacto de sus investigadores y la vinculación misma de la institución con su entorno, para complementar, de esta manera, las mediciones bibliométricas tradicionales u otras mediciones del entorno de la universidad, como lo considera, por ejemplo, el Manual de Valencia.

Palabras Clave: Altmetrics. Impacto. Investigadores. Plataformas académicas. Universidad. Visibilidad.

\begin{abstract}
This work is the result of one of the components of a macro investigation, which seeks to develop a Model to identify the degree of linkage of a university with its environment; in this case, it is applied to the University of Antioquia (Medellín-Colombia). This component refers to the visibility and impact of investigators from the perspective and data offered by altmetrics. To that effect, the altmetrics data of 1,032 investigators from six areas of knowledge are reviewed, taking into account different academic, professional and social platforms, as well as an integrating platform of altmetrics indicators. Although this measurement refers to University of Antioquia investigators, the

\footnotetext{
1 Universidad de Antioquia, Escuela Interamericana de Bibliotecología. Cl.67\#53-108, Blq. 12-305, Medellín, Colombia. Dirección de correspondencia/ Correspondence to: A. Uribe-Tirado. E-mail: <alejandro.uribe2@udea.edu.co>.

Recibido el 1 del abril del 2019, versión final presentado el 14 del agosto del 2019 y aprobado el 19 del septiembre del 2019.

Como citar este artículo/How to cite this article

URIBE-TIRADO, A. et al. Visibilidad e impacto altmétrico de los investigadores de la Universidad de Antioquia: metodología aplicable a universidades. Transinformação, v.31, e190016, 2019. http://dx.doi.org/10.1590/2318-0889201931e190016
} 
general methodology for data capture and interpretation may be extended to other universities that share the same investigation and scientific communication, and that from the altmetrics wish to identify the visibility and impact of their investigators and the linking of the institution with its environment, and complement in this way, the traditional bibliometric measurements or other measurements of the university environment, such as the Manual of Valencia.

Keywords: Altmetrics. Impact. Researchers. Academic platforms. University. Visibility.

\section{Introducción}

La pregunta referente a la ciencia y la relación con las comunidades académicas y no académicas ha generado la necesidad de comprender las diferentes formas de producción de conocimiento. Las universidades e instituciones de investigación son vistas como actores fundamentales en la creación de conocimiento de cara a la solución de necesidades sociales. Lo anterior ha implicado cambiar la forma de estudiar la producción de conocimiento, pasando de analizar solo los esfuerzos centrados en las comunidades académicas a comprender el relacionamiento con la misma sociedad (Gibbons et al.,1994).

En este contexto, como lo menciona Fleet et al. (2017), los roles de la academia vienen experimentando diversas transformaciones, combinando diferentes lógicas y visiones que permitan responder al entorno productivo y social. Esto demuestra la importancia de ampliar la comprensión del accionar de la ciencia a partir del intercambio de conocimientos con comunidades y no solo de lo que esta puede entregar de manera unidireccional. Esta perspectiva se plantea como el concepto de vinculación, el cual implica cambiar a una lógica de relacionamiento multidireccional que deben tener las universidades e instituciones de investigación con su entorno.

Lo anterior pone de manifiesto nuevos retos para los procesos de comunicación y uso del conocimiento científico, pues, si se suma a la masificación e integración constante de las tecnologías de la Web social, la visión desde la vinculación hace necesario pensar en la visibilidad y el impacto que genera la investigación, no solo en las comunidades científicas, sino en la sociedad misma, lo que convierte en un imperativo para las universidades, grupos de investigación e investigadores, la exploración y uso de nuevos medios y métricas.

Todo ello hace pensar en la importancia de ampliar la comprensión de los conceptos de visibilidad e impacto, entendiendo que este último no se puede reducir al ámbito de las comunidades científicas. También es importante analizarlo con relación a otros sectores y grupos de la sociedad, como lo muestra Bastow et al. (2014) al identificar que hay un nivel de impacto tradicional, pero que hay otros niveles (medio y externo) que implican a la sociedad - Impacto social. Al respecto, Alperin (2014), desde la perspectiva de las publicaciones científicas, define el impacto como:

[...] el grado al que los estudios publicados en revistas académicas son aplicados, citados, discutidos o han de alguna otra forma afectado a individuos y/o grupos dentro o más allá de las comunidades académicas. Esto es, realizamos una distinción entre acceso (esto es, alcance) y uso (esto es, impacto), siendo el primero un prerrequisito del segundo. Medir el impacto es ciertamente un objetivo escurridizo (especialmente el impacto social) (Alperin, 2014, p.21).

Lo mencionado implica considerar los procesos de investigación en relación con el entorno, y, por tanto, con los diferentes sectores sociales, lo que conlleva a pensar en el compromiso de visibilizar y analizar, tanto el impacto científico como social generado, directo o indirecto. Por tanto, es necesario considerar la visibilidad académica y científica (Uribe-Tirado, 2015) como un paso previo para lograr un mayor impacto social.

También es importante considerar que en regiones como América Latina una porción considerable de las actividades relacionadas con la investigación se comunica fuera de los medios académicos tradicionales, ya que, por ejemplo, Organizaciones no Gubernamentales y centros de investigación producen una gran cantidad de literatura 
de mayor relevancia para el ámbito local o regional, depositando los productos en repositorios digitales y redes sociales (Alperin, 2014; Erdt et al., 2016). Esto implica pensar en nuevas métricas que permitan analizar la visibilidad e impacto en esos medios y que complementen los indicadores tradicionales como, por ejemplo, la citación en bases de datos WOS y Scopus, el factor de impacto y el citation score, los cuales dificultan conocer el desarrollo de la ciencia y poder analizar los impactos que generan, sobre todo en el contexto latinoamericano. Por ello, como lo indica Alperin (2014, p.15), "existe una necesidad urgente de encontrar indicadores alternativos de producción de investigación que capturen una porción mayor de la producción de las regiones en desarrollo".

Dado el panorama anterior, en años recientes ha habido un interés creciente de la comunidad académica y científica en desarrollar mejores indicadores (Alperin, 2014; Tunger et al., 2018) que incluyan nuevos medios de comunicación de la ciencia y que permitan proyectarse en la perspectiva de vinculación. Ante esto, las altmetrics aparecen como una propuesta complementaria; sin embargo, se encuentran aún en un desarrollo inicial, especialmente cuando se las compara con los indicadores bibliométricos tradicionales, que llevan décadas de trabajo (Alperin, 2014).

Asimismo, es importante comprender la diferenciación que presenta Instituto Interuniversitario de Investigación Avanzada sobre Evaluación de la Ciencia y la Universidad (2014) que clarifica y actualiza la propuesta de Björneborn e Ingwersen (2004), en la cual la altmetrics (altmetría) es otra métrica que tiene aspectos comunes con la bibliometría, pero que no la incluye completamente; es decir, son metrías diferentes, pero se complementan.

Para este trabajo, a partir de investigaciones anteriores y del análisis de distintas definiciones, se entienden, en sentido general, las altmetrics (métricas complementarias) como:

El estudio y toma de decisiones en la actividad científica y académica, a partir de la creación y análisis de nuevos indicadores y fuentes de información y medición, basados especialmente en la Web social, que consideran el uso (acceso y descarga), la evaluación por pares (opinión de especialistas e interesados), las citaciones, y las interacciones y recomendaciones (almacenamiento, links, favoritos, conversaciones y comentarios) de diferentes publicaciones y contenidos por distintos canales, formatos y/o herramientas: formales-informales, tradicionales-actuales, físicos-digitales (Uribe-Tirado; Alhuay-Quispe, 2017, p.2).

Como tal, su trascendencia y beneficios aún están siendo explorados. Al respecto, Piwowar (2013) indica cuatro ventajas potenciales, a saber: mejor entendimiento del impacto, revelando cómo los productos académicos son leídos, discutidos, guardados y recomendados, así como citados; evidencia del impacto en días y no en años; imagen del impacto de los productos académicos creados para Internet como software, posts en blogs, videos, entre otros; conocimiento de la visibilidad e impacto en diferentes públicos, incluyendo académicos, profesionales, clínicos, educadores y el público en general.

Respecto a la literatura sobre el tema, ha aumentado considerablemente la publicación sobre altmetrics en los últimos años (Erdt et al., 2016; Tunger et al., 2018), principalmente se encuentran trabajos sobre el potencial de uso de estas métricas para la evaluación científica y la política científica (Weller, 2015; González-Valiente et al., 2016; Haustein, 2016; Tunger et al., 2018), la generación de nuevos indicadores (Torres-Salinas et al., 2013; Orduña-Malea; Martín-Martín; Delgado-López-Cózar, 2016), el análisis comparativo y de correlación con métricas tradicionales (Bar-Ilan, 2014; Bornmann, 2014; Costas et al., 2015; Karanatsiou et al., 2017; Uribe-Tirado; Alhuay-Quispe, 2017; Asemi; Heydari, 2018; Bornmann; Haunschild, 2018; Martin-Martin et al., 2018), la importancia de combinar y explotar datos de diversas fuentes de información (Thelwall et al., 2013; Erdt et al., 2016; Orduña-Malea; Martín-Martín, Delgado-López-Cózar, 2016; Sugimoto et al., 2017; Asemi; Heydari, 2018) y el funcionamiento de los agregadores altmétricos como Plum Analytics, Altmetric.com, ImpactStory, entre otros (Williams, 2017; Ortega, 2018). 
Como se verá, la mayoría de las métricas disponibles implican un nivel de interacción que cabría dentro de la definición de impacto presentada. Así, aunque una mención específica (esto es, en redes sociales, favoritos, o una entrada en un blog) es una indicación de uso, también provee información sobre la extensión del alcance y proporciona evidencia de uno de los canales por los cuales se diseminan los estudios (Alperin, 2014).

Para finalizar, es importante indicar, que algunos estudios muestran cómo cada vez hay un uso mayor por parte de los académicos de nuestros países de las redes sociales, de la Web 2.0, aunque sin ser todavía muy extendido en algunas regiones, pero con una tendencia creciente (de Araujo, 2014, 2017; Aguillo Cano; UribeTirado; López López, 2017). No obstante, cuando se hacen mediciones de altmetrics de manera directa usando alguno de los proveedores de estas métricas (Altmetrics.com, ImpactStory, etc.), los resultados son bajos en nuestra región latinoamericana (Alperin, 2015; Fausto et al. 2015), tal y como se evidencia en esta investigación, y por ello se hace necesario, como se verá a continuación, realizar la captura de datos desde cada plataforma y unirla con los datos aún escasos para nuestros contextos de las plataformas directas e integradoras de altmetrics, para así lograr un panorama más completo de las métricas en nuestros contextos, para este caso, en una universidad colombiana.

Este trabajo presenta los resultados del componente de Altmetrics de la investigación mencionada. Principalmente, se analiza la visibilidad y el impacto de 1.032 investigadores de la Universidad de Antioquia $(U d e A)^{2}$ en diferentes plataformas académicas y sociales y una herramienta integradora de datos altmétricos. Se crea una metodología general, a nivel de universidad, para capturar e interpretar esos datos, la cual facilita que pueda replicarse este tipo de medición en otras universidades que comparten características de investigación y de comunicación científica y que, a partir de las altmetrics, desean identificar la visibilidad e impacto de sus investigadores y la vinculación misma de la institución con su entorno.

\section{Procedimientos Metodologícos}

Los diversos entornos que se vinculan desde la producción científica, como se mencionó, según Bastow et al. (2014), implican no solo las comunidades científicas (tradicional academic), sino también otros públicos, otros entornos donde hay visibilidad e impacto de esa investigación, y por tanto vinculación, como lo es, para este caso, la presencia en plataformas sociales, además de la visibilidad en los medios de comunicación, la colaboración con las organizaciones sociales y de emprendimiento, los profesionales de diferentes disciplinas y las redes de trabajo (mediating middle), los políticos y entidades gubernamentales y el sector privado y empresarial-comercial (external society). Este artículo aborda la presencia en plataformas sociales, los demás elementos son objeto de análisis del proyecto de investigación mas no se desarrollan en este artículo.

A partir de la revisión de la literatura de altmetrics y de ciencia 2.0 (tema relacionado con estas nuevas métricas) realizada anteriormente, y considerando las fuentes de información Web utilizadas para identificar visibilidad e impacto altmétrico, especialmente las plataformas académicas, profesionales y/o sociales - plataformas horizontales o verticales (Ponce, 2012; Ollé; López-Borrull, 2017) - y las herramientas integradoras de altmetrics a partir del DOI de las publicaciones, se realizó una caracterización y, adicionalmente, un análisis de la información de altmetrics (https://tinyurl.com/y2ppgqfk).

Tras lo anterior, respecto a lo que se denomina plataformas, se tomaron en cuenta las siguientes:

- Research Gate (https://www.researchgate.net/).

- Academia.edu. (https://www.academia.edu/).

\footnotetext{
2 Universidad pública fundada en 1803 con varias sedes en el departamento (Provincia) de Antioquia-Colombia. Cuenta con 104 programas de estudio de pregrado y 177 de posgrado (especialidades médicas, especializaciones, maestrías y doctorados). Tiene alrededor de 40848 estudiantes matriculados y 7947 profesores. Actualmente, registra 272 grupos de investigación correspondientes a 1900 potenciales investigadores. A su vez, registra una producción anual promedio de 1100 artículos científicos, según datos de Scopus, Web of Science y Lens.
} 
- Mendeley (https://www.mendeley.com/).

- Google Scholar (perfiles) (https://scholar.google.es/).

- ORCID (https://orcid.org/).

- Autores Redalyc (https://www.redalyc.org/autorHome.oa).

- My Science Work (https://www.mysciencework.com/).

- Open Science Framework (https://osfio/).

- Linkedln (https://www.linkedin.com/).

- Facebook (https://www.facebook.com/).

- Twitter (https://twitter.com/).

Es importante tener en cuenta que, aunque Twitter ha sido una de las herramientas de las redes sociales más estudiadas en relación con la altmetría, esos estudios se han centrado en identificar la mención de artículos en esta red y la visibilidad e impacto que esto conlleva (Bornmann; Haunschild, 2016; Peoples et al., 2016). Sin embargo, para este estudio, el énfasis está en los perfiles de los investigadores en estas redes y, para el caso de Twitter, tras la prueba piloto realizada, se evidenciaron dificultades respecto a la identificación de las cuentas de los investigadores, ya que esta red se caracteriza por el uso de alias y no era fácil ubicar la cuenta y perfil de cada investigador en específico, por lo cual no se profundizó en ella de manera directa (se tomarían los datos que arrojara para esta herramienta, la plataforma Altmetrics.com), aunque se reconoce que es clave para la medición de altmetrics, como lo han indicado varios trabajos.

Respecto a datos altmétricos desde herramientas integradoras, se identificó que actualmente hay disponibilidad de varias opciones, algunas de acceso libre sin costo, pero otras con accesos limitados o que implicaban suscripción: Altmetrics.com (https://www.altmetric.com/), ImpactStory (https://impactstory.org/), PlumX Metrics (https://plumanalytics.com/), ALM-PLoS (https://www.plos.org/article-level-metrics), Kudos (https://www. growkudos.com/), etc.

En este caso se decidió, por experiencia en las etapas previas de esta investigación, utilizar Altmetrics. com, su espacio de Altmetrics Explorer y su API (https://www.altmetric.com/products/free-tools/). Tras elegir las plataformas y la herramienta de altmetrics a utilizar, se realizó un proceso metodológico dividido en 3 grandes etapas.

\section{1) Normalización de los nombres de los investigadores (1032 UdeA)}

La normalización de nombres es una actividad importante para la identificación de perfiles en las plataformas tenidas en cuenta. Se tomó el listado de los nombres $\mathbf{3}$ y, por medio de Excel, se separaron los datos creando diferentes columnas con el primer nombre, el segundo nombre, el primer apellido y el segundo apellido, con el fin de crear diferentes fórmulas de búsqueda de perfiles, agregando además datos tales como facultad, departamento, grupo de investigación, con el fin de identificar homónimos.

El listado general de los investigadores de la Universidad de Antioquia se organizó por facultades y se normalizaron los nombres y apellidos de los investigadores para facilitar la búsqueda. Esto llevó finalmente a la normalización de una muestra de 1,032 investigadores que se analizaron en esta investigación, distribuidos por facultades: Facultad de Medicina (419), Facultad de Ingeniería (189), Facultad de Ciencias Exactas y Naturales (181), Facultad de Ciencias Sociales y Humanas (108), Facultad de Ciencias Agrarias (68) y Facultad de Artes (67).

\footnotetext{
${ }^{3}$ Este listado fue suministrado por la Vicerrectoría de Investigación de la Universidad de Antioquia que ellos directamente normalizan y confirman teniendo en cuenta la identificación de los investigadores en el CVLAC (Hoja de vida de los investigadores para el Sistema Nacional de Ciencia, Tecnología e Innovación - COLCIENCIAS) y aquellos que tienen ORCID.
} 


\section{2) Revisión y recopilación de datos de plataformas}

\section{- Identificación y selección de la información altmétrica ofrecida por cada plataforma}

El formato de recolección de datos (https://tinyurl.com/y2ppgqfk), contiene tanto indicadores de caracterización de los autores que son comunes para todas las plataformas consideradas, como: nombres, primer apellido, segundo apellido, facultad, si tiene perfil en la plataforma, $n^{\circ}$ de perfiles, nombre en el perfil, etc. En la siguiente Figura 1 se presenta una síntesis de los indicadores altmétricos específicos de cada plataforma.

\section{- Utilización de API's de las plataformas que lo permiten}

Para la recopilación de datos, se utilizaron dos tipos de búsquedas: manual y por medio de APIs (Interfaz de Programación de Aplicaciones). En ambos casos, fueron adaptadas y sistematizadas por el equipo de investigación de este proyecto, posibilitando la extracción de los datos que se requerían, y así, ahorrando tiempo en búsquedas y extracción de información según las posibilidades que ofrecía cada plataforma. Las plataformas que aprovecharon las API's fueron Research Gate y Google Scholar; para el resto de las plataformas exploradas se hizo de forma manual
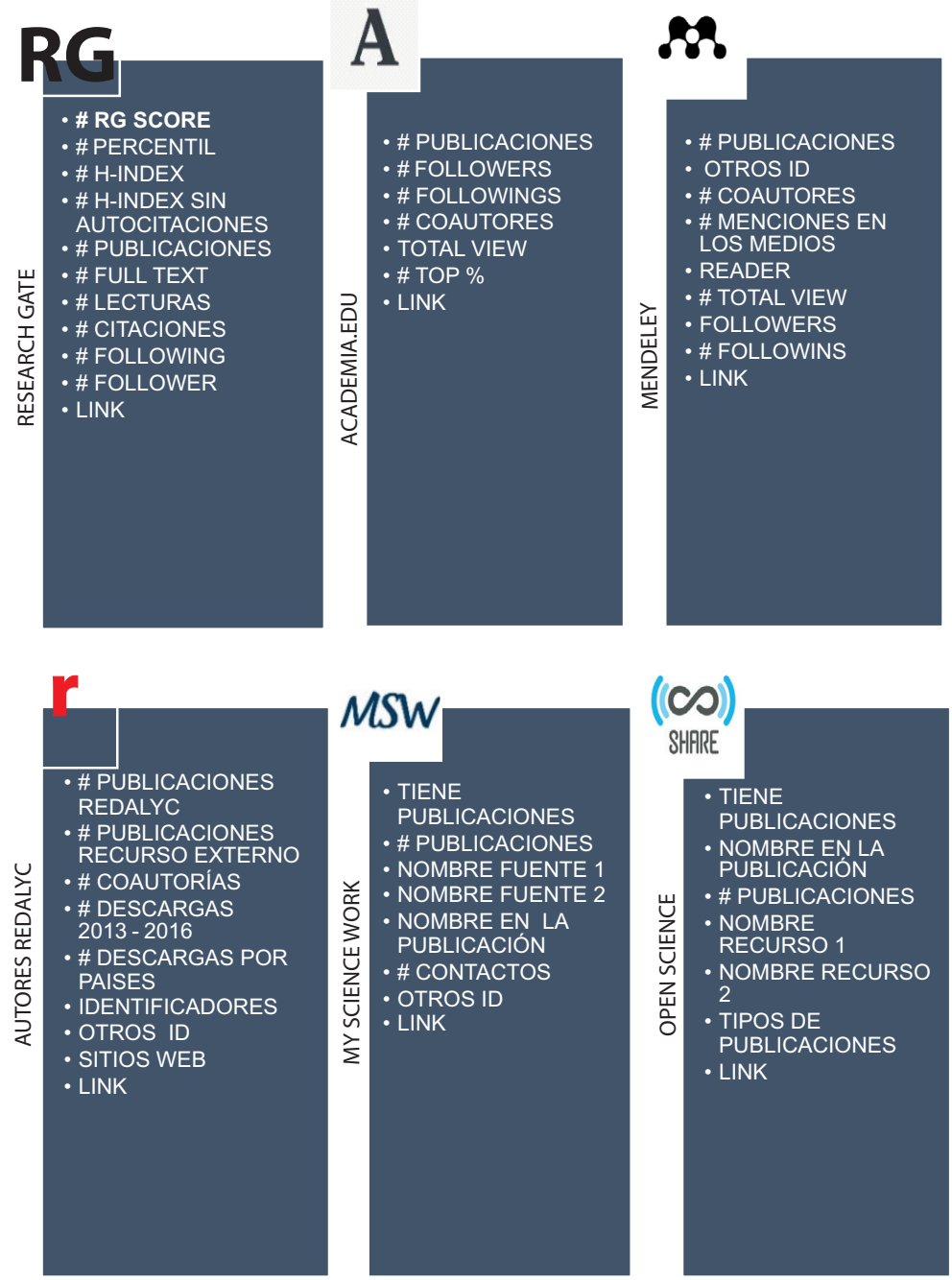


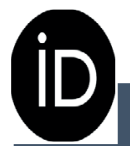

- NOMBRE EN E PERFIL

- AÑO DE INICIO

- OTROS ID

-\#

PUBLICACIONES

음 - LINK
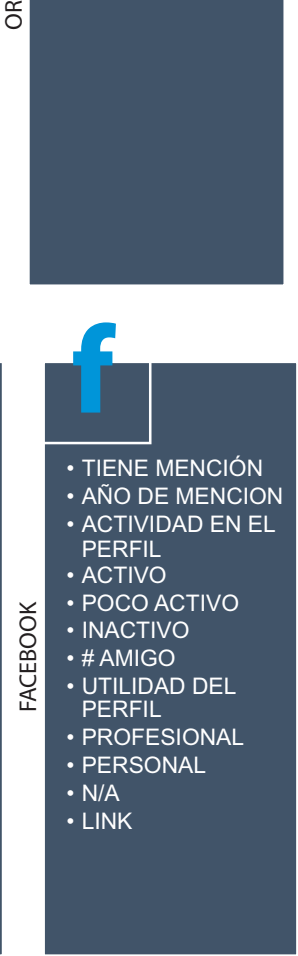

Figura 1. Indicadores altmétricos recopilados.

Fuente: Elaboración propia (2018) 
debido a que la seguridad de éstas bloqueaba la extracción automática o no facilitaba todos los datos de interés según los indicadores altmétricos identificados.

- Prueba piloto y cálculo de tiempos de recogida de información

Se realizó una prueba piloto para aquellas plataformas que implicaban un trabajo manual para calcular el tiempo de búsqueda y recolección de información de los perfiles de los investigadores. Esta se aplicó a una muestra del $15 \%$ de los investigadores, proporcional a la cantidad de investigadores según las Facultades y áreas de conocimiento. Los resultados obtenidos identificaron que la búsqueda manual de los perfiles de los investigadores en las diferentes plataformas osciló entre 3 a 5 minutos aproximadamente, esto se debe a que el nombre del investigador requiere de algunas variaciones como: 1 nombre +1 er apellido $+2^{\circ}$ apellido, pero gracias a su combinación fue posible identificar al investigador requerido y realizar la búsqueda de los perfiles de forma completa. La recogida de información puede tardar entre 3 a 6 minutos aproximadamente, dependiendo de la cantidad de datos que contenga el perfil y la especificidad de la información que se requiere para consolidar los indicadores altmétricos.

- Rastreo y registro manual de los datos de las plataformas que no permiten APl o cuyo funcionamiento no permite la recogida de información clave y específica de altmetrics.

Después de tener organizados los indicadores altmétricos específicos para cada plataforma, se procede a hacer la búsqueda y recogida de información de los perfiles en el tiempo estimado y posteriormente se realiza el análisis de los datos obtenidos.

\section{3) Obtención de datos de una herramienta integradora de altmetrics}

- Rastreo de la publicación de los investigadores desde Google Scholar e identificación en dicha producción de los Direct Object Identifier (DOI): Para el caso de los 1.032 investigadores de la UdeA se identificaron, a partir de 2006 y hasta 2018, un total de 15.504 artículos, de los cuales presentaban DOI 7790 (50,2\%).

- Recogida de información desde la APly confirmación con Altmetrics Explorer: Este proceso se realizó la segunda semana de enero de 2019, buscando tener todos los DOI disponibles de autores de la UdeA hasta 2018.

Finalmente, seguidas estas 3 etapas y los diferentes procesos, fue posible la recogida de información para este caso de investigadores UdeA, pero, a su vez, la validación de esta metodología para ser utilizada por cualquier otra universidad que desee tener una mirada amplia de sus investigadores considerando distintos indicadores de altmetrics.

\section{Resultados}

A continuación, considerando las posibilidades de extensión de este texto y la gran cantidad de información recopilada, tanto desde las plataformas como desde cada uno de los artículos con DOI desde la herramienta Altmetrics.com, se presentan algunos datos generales, con diferencias según plataforma, como una muestra de las posibilidades de información altmétrica que una universidad puede obtener de sus investigadores; pero el objetivo principal de presentar algunos de estos resultados es motivar a otras universidades de la región a realizarlo teniendo como base una metodología disponible y validada.

\section{1) Plataformas}

En primer lugar, hay una información que es clave identificar en todas las plataformas y que es la relativa a si hay presencia con perfil o no de los investigadores de la universidad en cuestión.

Para el caso de la Universidad de Antioquia, considerando los 1.032 investigadores analizados, se encontró que a partir de la identificación de la presencia de los investigadores en cada plataforma (Tabla 1), es posible 
profundizar en los datos métricos de los perfiles que sí están presentes y dan acceso a diferentes datos, teniendo la posibilidad de que los mismos sean segmentados por las distintas facultades, lo cual es clave para identificar si hay diferencias disciplinarias en cuanto a la participación o no en estas plataformas (Tabla 1).

Tabla 1. Presencia de los investigadores de la Universidad de Antioquia en plataformas por facultad.

\begin{tabular}{|c|c|c|c|c|c|c|c|c|c|c|c|c|c|c|}
\hline \multirow{2}{*}{ Plataforma } & \multicolumn{7}{|c|}{ Con perfil (\%) } & \multicolumn{7}{|c|}{ Sin perfil (\%) } \\
\hline & Art. & $\mathrm{CSH}$ & Agr. & Ingen. & Exact. & Med. & Total & Art. & $\mathrm{CSH}$ & Agr. & Ingen. & Exact. & Med. & Total \\
\hline Research Gate & 1 & 3 & 3 & 10 & 7 & 13 & 38 & 5 & 7 & 4 & 8 & 10 & 27 & 62 \\
\hline ORCID & 1 & 4 & 2 & 6 & 6 & 16 & 36 & 6 & 6 & 4 & 12 & 11 & 25 & 64 \\
\hline Linkedln & 1 & 3 & 2 & 8 & 5 & 14 & 32 & 5 & 7 & 5 & 10 & 13 & 27 & 68 \\
\hline Academia.edu & 2 & 6 & 2 & 4 & 4 & 7 & 24 & 5 & 5 & 4 & 15 & 13 & 34 & 76 \\
\hline Facebook & 3 & 3 & 2 & 5 & 3 & 9 & 24 & 4 & 8 & 5 & 14 & 14 & 32 & 76 \\
\hline Google Scholar & 0 & 3 & 1 & 5 & 5 & 5 & 19 & 6 & 7 & 6 & 14 & 13 & 36 & 81 \\
\hline Mendeley & 0 & 1 & 1 & 2 & 3 & 3 & 10 & 6 & 9 & 6 & 16 & 15 & 37 & 90 \\
\hline Autores Redalyc & 0 & 1 & 0 & 1 & 0 & 1 & 4 & 6 & 9 & 6 & 17 & 17 & 40 & 96 \\
\hline My Science Work & 0 & 0 & 0 & 0 & 0 & 0 & 0 & 6 & 10 & 7 & 18 & 18 & 41 & 100 \\
\hline Open Science Framework & 0 & 0 & 0 & 0 & 0 & 0 & 0 & 6 & 10 & 7 & 18 & 17 & 41 & 100 \\
\hline
\end{tabular}

Fuente: elaboración propia, 2019.

Nota: ART: Artículos; CSH: Ciencias Sociales y Humanas; Agr.: Ciencias Agrarias; Ingen: Ingeniería; Exact.: Ciencias Exactas y Naturales; Med.: Medicina.

Con ello, además de otros ejemplos de datos altmétricos que se presentan a continuación, se pretende tener resultados de mayor visibilidad e impacto, desde estas mismas plataformas o integrándose con datos bibliométricos, como lo han hecho diferentes estudios (Costas et al., 2015; Asemi; Heydari, 2018; Bornmann; Haunschild, 2018; Huang et al., 2018; Ortega, 2018), o como lo hará esta investigación en los próximos meses, al terminar el desarrollo de todos los componentes de este proyecto macro, integrando datos altmétricos como bibliométricos y otros relativos a la vinculación con el entorno de la Universidad de Antioquia a partir del Manual de Valencia, teniendo como punto de partida la Etapa 1 que se desarrolló para la Sede Universitaria de Investigación y 226 investigadores (Vélez et al., 2017).

Por ejemplo, de los diferentes datos altmétricos ofrecidos por Mendeley está el de las lecturas (Readers), el cual muestra la cantidad de lecturas que ha tenido el autor desde la plataforma y que, según estudios como Zahedi, Costas y Wouters (2017) y Maflahi y Thelwall (2018), es uno de los mayores previsores de futura citación (Ortega, 2018). Dándole una mirada a este indicador desde las facultades se puede encontrar el autor más visitado y leído en su perfil y probablemente citado, al igual que desde el área de conocimiento en la que converge, identificar la interacción y vinculación en esa plataforma social.

En este sentido, en el caso de la Universidad de Antioquia fue evidente que los perfiles y readers fueron más significativos en la Facultad de Medicina (perfiles: 31\%; readers: 16\%) y Ciencias Naturales (perfiles: 28\%; readers: 16\%) y mucho menos en las otras cuatro facultades de este estudio: Facultad de Ingeniería (perfiles: 21\%; readers: 11\%), Facultad de Ciencias Sociales (perfiles: 12\%; readers: 4\%), Facultad de Ciencias Agrarias (perfiles:7\%; readers: 3\%), y Facultad de Artes (perfiles: 2\%; readers: 0\%), lo cual permite identificar que no todas las áreas y disciplinas tienen la misma dinámica y datos.

De otro lado, entre estos datos altmétricos posibles de identificar, para toda una universidad o por facultades, es importante destacar algunos, como son los followers y following (seguidores y seguidos). En este caso, las plataformas Research Gate, Academia.edu y Mendeley utilizan el indicador de followers y following (seguidores 
y seguidos), y por medio de estos se puede identificar la popularidad del autor en el medio (Figura 2). En otras plataformas (Linkedln, My Science Work, entre otras), este identificador se puede encontrar de una forma más sencilla, como contactos, sin hacer la diferencia del seguido o seguidor.

Por otra parte, como lo han identificado distintos estudios (Martín-Martín et al., 2018), en la actualidad Google Scholar es una plataforma clave para entender la comunicación científica, para identificar la visibilidad y el impacto desde una perspectiva más amplia e integradora, que ha ido desplazando a las tradicionales WoS y Scopus.

Por tanto, para una universidad, es clave saber qué tanta presencia hay de sus investigadores en el perfil de Google Scholar y los datos métricos que reporta la Tabla 2, ya que se pueden relacionar con los datos de otras plataformas, tanto bibliométricas tradicionales como de altmetrics.

\section{FOLLOWERS}

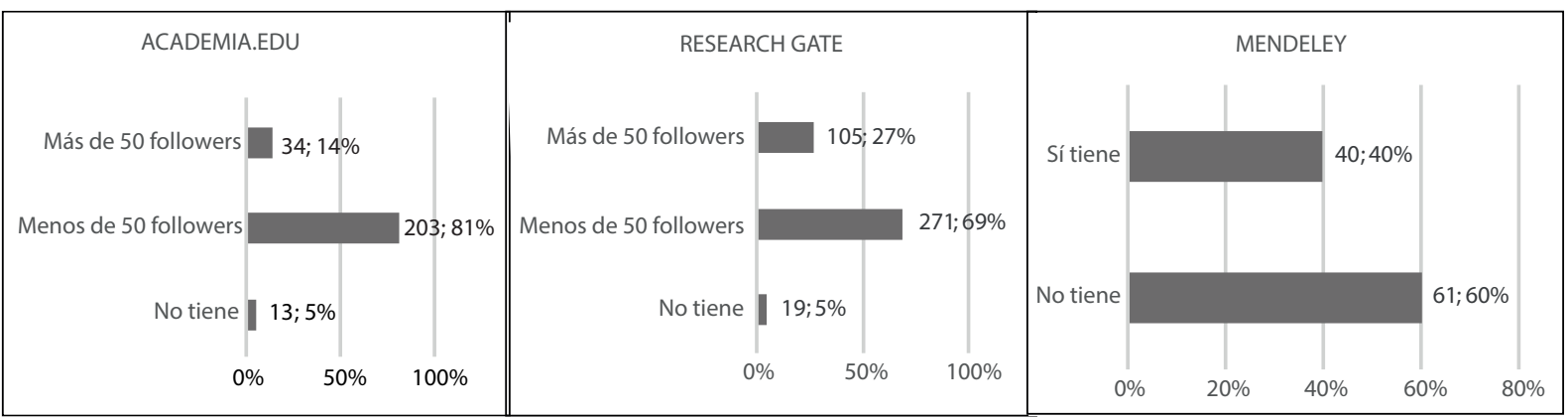

\section{FOLLOWINGS}

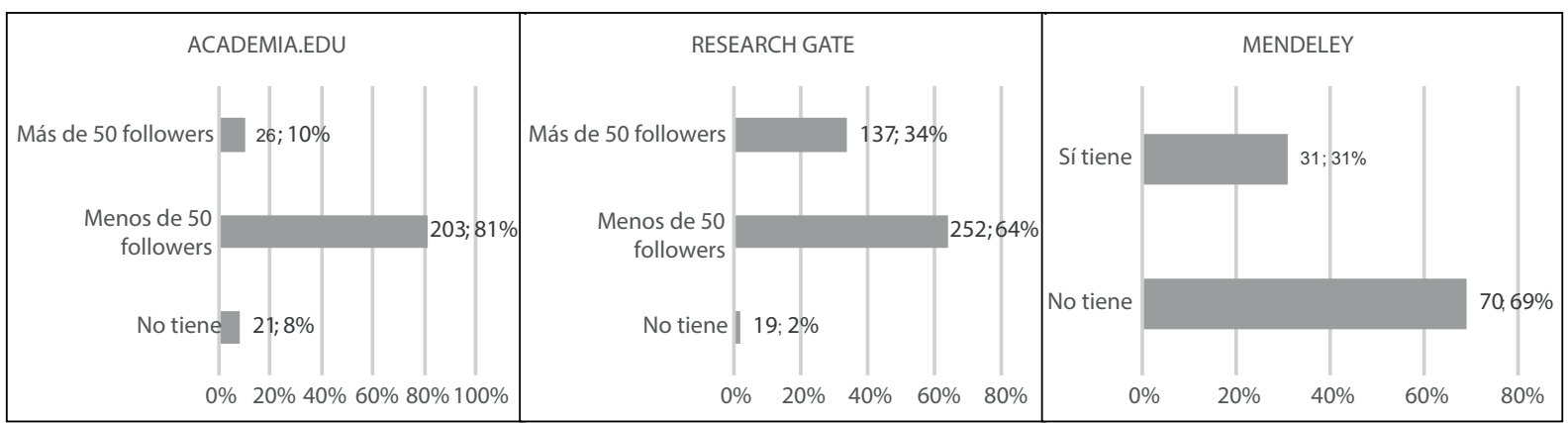

Figura 2. Ejemplo de followers y following en diferentes plataformas.

Fuente: Elaboración propia (2018).

Tabla 2. Comparativa de índices entre las facultades según datos de GS.

\begin{tabular}{lcccc}
\hline Facultad & Índice-H & i10 & Índice-H más alto & i10 más alto \\
\hline Facultad de Artes & 2 & 0 & 2 & 10 \\
Facultad de Ciencias Agrarias & 9 & 8 & 22 & 56 \\
Facultad de Ciencias Exactas y Naturales & 50 & 50 & 34 & 122 \\
Facultad de Ciencias Sociales y Humanas & 35 & 23 & 66 & 55 \\
Facultad de Ingeniería & 46 & 40 & 20 & 34 \\
Facultad de Medicina & 46 & 40 & 44 & 118 \\
\hline Totales & 188 & 161 & \\
\hline
\end{tabular}

Fuente: Elaboración propia (2018). 
A su vez, profundizar y valorar los datos que ofrecen estas plataformas es fundamental para que una universidad vaya más allá de si están o no presentes.

Seguidamente, se presentan algunos ejemplos para el caso de la Universidad de Antioquia, como el referente a la importancia de tener ORCID, ya que es un identificador de nombre que se ha convertido en estándar. Pero no basta con tener el número para poder colocarlo en un artículo o en una información que pida un organismo de ciencia universitario o nacional. Para aprovechar todo su potencial es necesario gestionar realmente ese perfil que implica el ORCID, lo cual desafortunadamente no es lo más común ni se hace de forma adecuada.

Es decir, muchos investigadores no tienen ORCID (caso UdeA: 64\%, 664 investigadores), y entre quienes sí lo tienen (Caso UdeA: 33\%, 368 investigadores), se estableció que la gestión de los perfiles de ORCID ${ }^{4}$ era Alta de parte de 123 investigadores (33\%), Media de 51 investigadores (14\%) y Baja de 194 investigadores (53\%), por lo que se está desaprovechando todo el potencial de visibilidad e impacto y de tener datos directos, tanto como investigador como para toda la universidad.

Sin embargo, es posible profundizar no solo en el nivel de gestión en una plataforma de origen académico como ORCID, sino que es posible también hacerlo en plataformas de un origen más social, pero que se están aprovechando para un uso académico, como lo es Facebook, a través de sus perfiles y la interacción con Grupos y Páginas de interés académico. Por ejemplo, además de identificar si los investigadores tienen presencia en esta red, analizar el tipo de presencia y qué tan activos o no son allí. Esto mismo es posible en otras plataformas, en este caso de perfil más profesional, como Linkedln (Figura 3).

Plataforma: Facebook
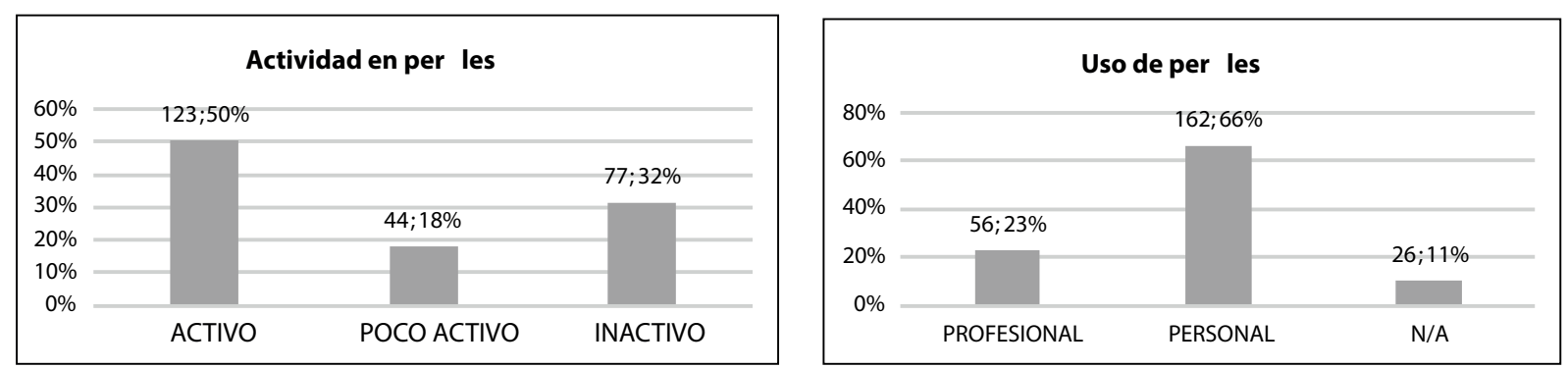

Plataforma: Linkedln
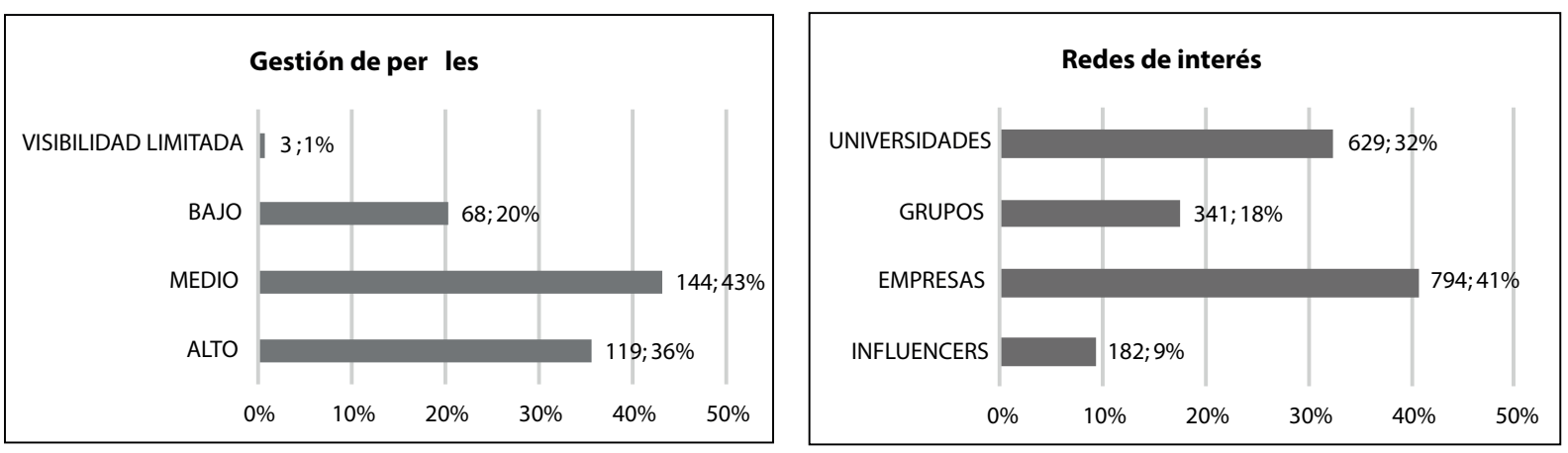

Figura 3. Tipos de actividad desde Facebook y Linkedln.

Fuente: Elaboración propia (2018).

${ }^{4}$ Para un mejor análisis de las plataformas ORCID, Facebook y Linkedin se vio necesario no solo quedarse con un análisis cuantitativo de los datos que
estas plataformas facilitaban, sino que se quiso profundizar más desde un análisis cualitativo (análisis de contenido) que permitiera identificar el grado
de gestión de los perfiles y la interacción desde lo académico que los investigadores tenían. Por tanto, se establecieron los niveles de gestión: activo,
poco activo, inactivo; de tipo de uso: profesional, personal, no identificablen/a; y de visibilidad: limitada, baja, media y alta y de interés: universidades, grupos,
empresas, influencers. 
De otra parte, estas plataformas permiten profundizar en la producción científica que reportan y desde allí extraer datos métricos importantes como, por ejemplo, en el caso de RedAlyc, y lo que se reporta desde Autores RedAlyc, que puede hacerse para autores para cada universidad. Para la Universidad de Antioquia hay 283 investigadores, y para estos se reportan datos claves que se pueden cruzar con las propias variables de esta plataforma y también de otras (https://www.redalyc.org/institucion.oa?id=15344\&tipo=autores).

\section{2) Herramienta Altmetrics}

Como se indicó anteriormente, la información de las plataformas sociales se centraba principalmente, y en primer lugar, en si el investigador tenía presencia o no, y desde los datos de cada una de estas, qué datos altmétricos era posible obtener. Pero a partir de lo señalado, este texto más que presentar todos los datos recopilados y posibles para la Universidad de Antioquia, lo que quiere presentar es la posibilidad que se pueden obtener más datos de un investigador-universidad y su producción que los que tradicionalmente ha dado la bibliometría (índice H, Factor de Impacto) y que, por tanto, estas plataformas y las altmetrics pueden facilitarnos esa mirada complementaria.

Por tanto, se presentan a continuación los datos de una herramienta integradora como Altmetric.com, analizando la producción de estos 1032 investigadores, como evidencia de las posibilidades de recopilar este tipo de datos que brindan este tipo de plataformas individualmente, para luego complementarlos.

Para este caso, después de hacer un rastreo bibliométrico (otro componente de este macroproyecto) en diferentes bases de datos, tanto comerciales (WoS y Scopus) como de acceso abierto (SciELO, RedAlyc) e integrándose con datos de Google Scholary Crossref, fue posible detectar una producción de 15.504 artículos para estos 1032 investigadores, entre 2006 y 2018.

Tras hacer el rastreo y depuración de los mismos, a través de la adaptación de API's, se detectó que para este universo (15.504 artículos), un total de 7.790 presentaban DOI (50,2\%) y a partir de los cuales era posible entonces, utilizando la herramienta altmétrica seleccionada (Altmetric.com), extraer distintos datos altmétricos de esta producción, como lo indica el sitio oficial de esta herramienta, referidos a: "Public policy documents, Mainstream media, Online reference managers, Post-publication peer-review platforms, Wikipedia, Open Syllabus Project, Patents, Blogs, Citations, Research highlights, Social Media" (Altmetrics.com, 2019).

Entre estos 7.790 artículos con DOl, reportaron algún dato altmétrico un total de 2.106 (27.0\%). Entre estos 2.106 artículos, un porcentaje del 84.6\% (1781) presentaba un AltmetricsScore menor a 10 y solo un 15.4\% (325) mayor a 10.

Cada uno de estos artículos es posible analizarlo o dividirlo por las 6 áreas que este proyecto trabaja, y así detectar diferencias disciplinarias o, en su defecto, dividirlos por año y detectar aspectos interesantes o previsiones de citación en el tiempo, como lo refiere Ortega (2018, p.587) al concluir que: "Los resultados han demostrado que las métricas de mención (Tweets y Menciones en blogs) son las primeras en aparecer, seguidas de las métricas de uso (Descargas y Vistas) y de marcadores (Lectores), y finalmente, los indicadores bibliométricos (Citas)".

\section{Conclusión}

\section{Respecto a la Universidad de Antioquia}

La clave del éxito para replicar y ejecutar un análisis altmétrico radica en la capacidad de adaptación que tenga la universidad al considerar nuevos indicadores métricos, sumada a la composición de un equipo de trabajo multidisciplinario, además de la planeación y las decisiones que se tomen a partir de los datos métricos a integrar. 
Ante estos datos, mayoritariamente bajos en varias plataformas (Tabla 1), se recomienda que el primer paso del proceso sea la identificación de las necesidades manifiestas por los investigadores en términos de visibilidad e impacto, para así trabajar en común acuerdo con las dependencias de investigación (Vicerrectoría, Centros, Grupos) y el Sistema de Bibliotecas UdeA, en torno a las acciones de mejora, orientadas a la formación (cultura informacional y ciencia 2.0) y a la posterior gestión de la presencia en la Web (identidad digital), considerando estos resultados producto del análisis de los 1032 investigadores.

A su vez, este trabajo permite reconocer de manera particular en cuál plataforma social existe mayor concentración de determinada área del conocimiento, en vista de que no todas las áreas se visibilizan en todas las plataformas sociales de la misma manera, ya sea por motivos de poca formación en cultura informacional o por desconocimiento. Acorde con lo anterior, al identificar cuáles facultades necesitan más apoyo para la visibilidad científica, podrán también desarrollarse servicios y/o programas específicos para dichas áreas.

En ese sentido, los datos obtenidos para la UdeA brindan un diagnóstico de su visibilidad e impacto actual desde la perspectiva altmétrica (que no es única) que, aunque reciente en el mundo académico, está siendo cada vez más importante, pero con posibilidades de mejora; por ello, la necesidad de encaminarse como universidad en un trabajo de formación, gestión y medición.

Afortunadamente, en el caso de la Universidad de Antioquia, esta necesidad se está consolidando en un trabajo conjunto entre la Vicerrectoría de Investigación, el Sistema de Bibliotecas y la Escuela Interamericana de Bibliotecología, a partir de dos estrategias relacionadas y ya asumidas en su Plan de Desarrollo 2017-2022 y su Plan de Acción (específico de la actual rectoría 2018-2021) sobre: ciencia abierta - visibilidad e impacto científico y social (http://www.udea.edu.co/wps/portal/udea/ web/inicio/institucional/direccionamiento-estrategico/plandesarrollo).

\section{Respecto a los datos altmétricos}

En este aspecto, es necesario mencionar que existe una tendencia o patrón generalizado y replicado en cada una de las plataformas, en donde se evidencia el desborde de nombres, seudónimos, composiciones diferentes al nombre oficial, los cuales son utilizados y casi que imposibles de recuperar, al igual que perfiles repetidos o abandonados en diferentes plataformas, lo que causa confusión al usuario a la hora de buscar y/o consultar.

Esta situación puede deberse a la falta de conocimiento frente al tema de identidad digital del investigador en entornos web, por lo que es fundamental, no solo para la UdeA sino para todas las universidades y especialmente en América Latina, la formación en competencias informacionales necesarias para la investigación, en los diferentes grupos y centros de investigación adscritos a cada universidad.

Por otro lado, indicadores como el de gestión de perfiles es uno de los datos altmétricos de gran valor y utilidad para un investigador, por lo que, al hacer mayor uso de una plataforma, participar activamente en redes de interés y gestionar completamente los datos de contacto e información personal, tarde o temprano obtendrá mayor visibilidad de su labor, a partir de su producción científica. Asimismo, puede medirse desde la bibliometría, de las altmetrics o desde la apropiación social del conocimiento, ya que, en última instancia, todas son mediciones que dan cuenta de la vinculación de la universidad con su entorno.

En conclusión, las plataformas sociales ofrecen dos grandes posibilidades: de visibilizar y de medir impactos. Ambos se complementan y pueden ser útiles personal o institucionalmente. Son también una oportunidad para dar a conocer el trabajo que se realiza desde cualquier área del conocimiento y acercarlo a diversos públicos. Este acercamiento podrá ser medido con la utilización de diferentes métricas ofrecidas en plataformas y herramientas especialmente diseñadas para tal propósito. 
El reto está, para las universidades (y las entidades estatales de ciencia), en estar abiertos a estas nuevas métricas, debido a que la bibliometría tradicional no está teniendo en cuenta la inmediatez y oportunidades que el mundo de las tecnologías de la información está proporcionando.

Las altmetrics son clave como complemento para la toma de decisiones, detectar necesidades de formación en cultura informacional y ciencia 2.0, identificar la visibilidad e impacto desde la visión de vinculación con el entorno y lograr una mirada más integradora. Por eso, es necesario, no quedarse solo con la mirada tradicional, bibliométrica, ya que la comunicación científica hoy requiere de otros ángulos, integrando las diferentes herramientas y plataformas si se busca una identificación más completa de esta vinculación (visibilidad e impacto científico y social).

En síntesis, recopilar la información desde estas distintas plataformas es fundamental para tener una mirada más integral de las métricas de los investigadores y de la universidad misma, ya que, además de integrarlas, en cada una de ellas es posible profundizar en distintos aspectos, algo cada vez más común, como indica Silvia Martínez Martínez, en el prólogo del libro La medición en plataformas sociales:

Estamos ante una realidad en continua evolución en la que conviven los recursos de medición que ofrecen las propias plataformas sociales junto a otros de carácter externo [...] En definitiva, existen diferentes combinaciones y soluciones posibles en las que la elección debería estar especialmente condicionada por los objetivos fijados y las métricas que nos interese obtener y seguir [... . no existe una solución única, sino que en cada caso se tendrá que decidir, escoger una opción o buscar la combinación más acertada para obtener los datos que posteriormente serán interpretados y analizados (Martínez Martínez, 2017, p.13).

Es decir, se requiere una mirada que integre lo bibliométrico y lo altmétrico, para con ello tener una visión holística de la visibilidad y el impacto e identificar así todas las formas de vinculación con el entorno que tiene una universidad; cumplir su misión para con la sociedad y su contexto particular.

Este ejercicio altmétrico da cuenta de que el impacto de la investigación también se está observando desde otros ángulos y que puede hacerse en universidades de nuestro contexto latinoamericano que pueden integrarse con la bibliometría y con la vinculación con el entorno, como propone el Manual de Valencia.

Así, las altmetrics son un complemento fundamental, como se evidencia en el propósito del macroproyecto que enmarca este componente y este artículo:

Este proyecto pretende construir un modelo que permita caracterizar y evaluar el impacto de los centros de investigación de la Universidad de Antioquia a través del desempeño de sus grupos de investigación e investigadores. Para ello se revisarán fuentes de información de la universidad, fuentes de información en la web como Web of Science, Scopus, Google Scholar, Gruplac-Colciencias, Google News, plataformas de Altmetrics; y se adaptarán y construirán indicadores para: 1) la evaluación de impacto científico, 2) formación en investigación, 3) apropiación y divulgación social del conocimiento, 4) presencia en medios de comunicación y 5) actividades de vinculación con el entorno (Restrepo-Quintero et al., 2016, p.2).

Este modelo general de medir la vinculación con el entorno, y específicamente esta metodología, para tener en cuenta los datos altmétricos, es un aporte que se quiere que sea tenido en cuenta, utilizado, mejorado y criticado desde la recopilación y reconocimiento de datos altmétricos por otras universidades de América Latina, ya que aún hay pocos estudios y desarrollos en este sentido, pero se ha comenzado un camino regional, como lo evidenció el primer LATmetrics (https://www.latmetrics.com), y afortunadamente, ya se tienen proyectadas nuevas versiones de LATmetrics para los próximos años en Perú 2019 (https://easychair.org/cfp/2LATmetrics), Cuba 2020 y Colombia 2021: 
LATmetrics surge em um momento de efervescência tanto para a comunicação científica quanto para a geografia da ciência. Mídias sociais e outros espaços digitais têm sido cada vez mais usados por pesquisadores e instituições para o compartilhamento de suas pesquisas com a sociedade, mudando a forma como medimos o impacto social da produção acadêmica. Novas oportunidades e uma série de desafios se impõem aos países periféricos, visto que a cobertura e a qualidade dos dados sobre métricas alternativas não costumam ser compatíveis com as dinâmicas da comunicação científica desenvolvidas nesta região (LATmetrics, 2018).

\section{Referencias}

Aguillo Cano, I.F., Uribe Tirado, A.; López López, W. Visibilidad de los investigadores colombianos según sus indicadores en Google Scholar y ResearchGate: Diferencias y similitudes con la clasificación oficial del sistema nacional de cienciaCOLCIENCIAS. Revista Interamericana de Bibliotecología, v.40, n.3, p.221-230, 2017. Disponible en: https://aprendeenlinea. udea.edu.co/revistas/index.php/RIB/article/view/327845. Acceso en: jul. 15, 2019.

Altmetrics.com (2019). Sources of Attention: Altmetric track a unique range of online sources to capture the conversations relating to research outputs. Available from: https://www. altmetric.com/about-our-data/our-sources/. Cited: Jul. 15, 2019.

Alperin, J.P. Indicadores de acceso abierto. Evaluando el crecimiento y uso de los recursos de acceso abierto de regiones en desarrollo: El caso de América Latina. In: Alperin, J.P. et al. Indicadores de acceso abierto y comunicaciones académicas en América Latina. Buenos Aires: Clacso, 2014. p.15-86. Disponible en: http://biblioteca.clacso.edu.ar/clacso/se/20141217052547/ Indicadores_de_acceso_abierto.pdf. Acceso en: Enero 31, 2019.

Alperin, J.P. Geographic variation in social media metrics: an analysis of Latin American journal articles. Aslib Journal of Information Management, v.67, n.3, p.289-304, 2015. Available from: https://stacks.stanford.edu/file/druid:sr068mj0031/Alperin GeographicVariationAltmetrics.pdf. Cited: Jul. 15, 2019.

Asemi, A., Heydari, M. Correlation between the articles citations in Web of Science (WoS) and the Readership Rate in Mendeley and Research Gate (RG). Journal of Scientometric Research, v.7, n.3, p.145-152, 2018. Doi: http://dx.doi.org/10. 5530/jscires.7.3.25

Bar-llan, J. Astrophysics publications on arXiv, Scopus and Mendeley: A case study. Scientometrics, v.100, n.1, p.217-225, 2014. Doi: http://dx.doi.org/10.1007/s11192-013-1215-1

Bastow, S. et al. The impact of the social sciences: How academics and their research make a difference. London: Sage, 2014. Available from: https://uk.sagepub.com/en-gb/ eur/the-impact-of-the-social-sciences/book241492. Cited: Jan. 31, 2019.

Björneborn, L.; Ingwersen, P. Toward a basic framework for webometrics. Journal of the American Society for Information Science and Technology, v.55, n.14, p.1216-1227, 2004. Doi: http://doi.org/10.1002/asi.20077

Bornmann, L. Alternative metrics in scientometrics: A metaanalysis of research into three altmetrics. Scientometrics, v.103, n.3, p.1123-1144, 2014. Doi: http://dx.doi.org/10.1007/ s11192-015-1565-y

Bornmann, L.; Haunschild, R. t factor: A metric for measuring impact on Twitter. Malaysian Journal of Library \& Information Science, v.21, n.2, p.13-20, 2016. Available from: https://
ejournal.um.edu.my/index.php/MJLIS/article/view/1708. Cited: Jul. 15, 2019.

Bornmann, L.; \& Haunschild, R. Do altmetrics correlate with the quality of papers? A large-scale empirical study based on F1000Prime data. PLoS One, v.13, n.5, p.1-12, 2018. Doi: http:// dx.doi.org/10.1371/journal.pone.0197133

Costas, R. et al. Do "altmetrics" correlate with citations? Extensive comparison of altmetric indicators with citations from a multidisciplinary perspective: Do "Altmetrics" Correlate With Citations? Journal of the Association for Information Science and Technology, v.66, n.10, p.2003-2019, 2015. Doi: http://dx.doi.org/10.1002/asi.23309

de Araujo, R.F.Ciência 2.0 e a presença online de pesquisadores: visibilidade e impacto. Ciência da Informação em Revista, v.1, n.3, p.32-40, 2014. Disponivel em: http://www.seer.ufal.br/ index.php/cir/article/view/1608. Acesso em: 15 jul. 2019.

de Araujo, R.F. Presença online de pesquisadores na web: indícios para as métricas em nível de autores. Pesquisa Brasileira em Ciência da Informação e Biblioteconomia, v.12, n.2, p.202-211, 2017. Disponivel em: http://repositorios.questoesemrede.uff. br/repositorios/handle/123456789/3560. Acesso em: 15 jul. 2019.

Erdt, M. et al. Altmetrics: An analysis of the state-of-the-art in measuring research impact on social media. Scientometrics, v.109, n.2, p.1117-1166, 2016. Doi: http://dx.doi.org/10.1007/ s11192-016-2077-0

Fausto, S. et al. Does the Global South have altmetrics? Analyzing a Brazilian LIS journal. In: Proceedings of ISSI. Istanbul, 2015. Available from: https://bdpi.usp.br/item/002766460. Cited: Jul. 15, 2019.

Fleet, N. et al. Midiendo la vinculación de las instituciones de educación superior con el medio y su impacto: Estudio de las mejores prácticas en el mundo y desarrollo de un instrumento piloto para instituciones chilenas. Cuadernos de Investigación, n.6, 2017. Disponible en: https://www.researchgate.net/ publication/319532060_Midiendo_la_vinculacion_de_las_ instituciones_de_educacion_superior_con_el_medio_y_ su_impacto_Estudio_de_las_mejores_practicas_en_ el_mundo_y_desarrollo_de_instrumento_piloto_para_ instituciones_chilenas. Acceso en: Enero 23, 2019.

Gibbons, M. et al. The new production of knowledge: The dynamics of science and research in contemporary societies. London: Sage, 1994. Available from: https://uk.sagepub.com/ en-gb/eur/the-new-production-of-knowledge/book204307. Cited: Jul. 23, 2019.

González-Valiente, C.L. et al. A review of altmetrics as an emerging discipline for research evaluation. Learned 
Publishing, v.29, n.4, p.229-238, 2016. Doi: http://dx.doi.org/10. 1002/leap.1043

Haustein, S. Grand challenges in altmetrics: heterogeneity, data quality and dependencies. Scientometrics, v.108, n.1, p.413-423, 2016. Doi: http://dx.doi.org/10.1007/s11192-016$1910-9$

Huang, W. et al. A correlation comparison between altmetric attention scores and citations for six PLOS journals. PloS One, v.13, n.4, 2018. Doi: http://dx.doi.org/10.1371/journal. pone.0194962

Instituto Interuniversitario de Investigación Avanzada sobre Evaluación de la Ciencia y la Universidad. Altmetría, influmetría... jInformetría! 2014. Madrid: Universidad Autónoma de Madrid y Universidad Carlos III de Madrid, 2014. Disponible en: http://www.inaecu.com/altmetria-influmetriainformetria/. Acceso en: jul.15, 2019.

Karanatsiou, D. et al. Bibliometrics and altmetrics literature review: Performance indicators and comparison analysis. Performance Measurement and Metrics, v.18, n.1, p.16-27, 2017. Doi: http://dx.doi.org/10.1108/PMM-08-2016-0036

LATmetrics. Anales. Niterói: Universidade Federal Fluminense, 2018. Disponible en: https://app.uff.br/riuff/bitstream/1/810 4/1/ANAIS\%20DO\%201\%20LATMETRICS.pdf. Acceso en: feb. 1,2019.

Maflahi, N.; Thelwall, M. How quickly do publications get read? The evolution of Mendeley reader counts for new articles. Journal of the Association for Information Science and Technology, v.69, n.1, p.158-167, 2018. http://doi.org/10.1002/ asi.23909

Martín-Martín, A. et al. Google Scholar, Web of Science, and Scopus: A systematic comparison of citations in 252 subject categories. Journal of Informetrics, v.12, n.4, p.1160-1177, 2018. Doi: http://dx.doi.org/10.1016/j.joi.2018.09.002

Martínez Martínez, S. Prólogo. In: Segovia, A. La medición en plataformas sociales. Barcelona: Editorial UOC-EPI, 2017. Disponible en: http://www.editorialuoc.cat/la-medicion-enplataformas-sociales. Acceso en: Enero 20, 2019.

Ollé, C.; López-Borrull, A. Redes sociales y Altmetrics: nuevos retos para las revistas científicas. In: Revistas científicas: situación actual y retos de futuro. Barcelona: Edicions Universitat de Barcelona, 2017. p.197-219. Disponible en: http://hdl.handle.net/10609/76227. Acceso en: Enero 25, 2019.

Orduña-Malea, E.; Martín-Martín, A.; Delgado-López-Cózar, E. The next bibliometrics: ALMetrics (Author Level Metrics) and the multiple faces of author impact. El Profesional de la Información, v.25, n.3, p.485-496, 2016. Doi: http://dx.doi. org/10.3145/epi.2016.may.18

Ortega, J.L. The life cycle of altmetric impact: A longitudinal study of six metrics from PlumX. Journal of Informetrics, v.12, n.3, p.579-589, 2018. Doi: http://dx.doi.org/10.1016/j.joi.2018. 06.001

Peoples, B.K. et al. Twitter predicts citation rates of ecological research. PloS One, v.11, n.11, e0166570, 2016. Available from: https://journals.plos.org/plosone/article?id=10.1371/journal. pone.0166570. Cited: Jul. 15, 2019.

Piwowar, H. Introduction altmetrics: What, why and where? Bulletin of the American Society for Information Science and
Technology, v.39, n.4, p.8-9, 2013. Doi: http://dx.doi.org/10.10 02/bult.2013.1720390404

Ponce, I. Monográfico: redes sociales: clasificación de redes sociales. Disponible en: http://recursostic.educacion.es/ observatorio/web/es/internet/web-20/1043-redes-sociales? start=3. Acceso en: Enero 23, 2019.

Restrepo-Quintero, D. et al. Impacto social, científico y actividades de vinculación con el entorno de centros de investigación de la Universidad de Antioquia. Medellin (Colômbia): Universidad de Antioquia, 2016. Convocatoria Programática 2016, Área Ciencias Sociales, Humanidades y Artes. Disponible en: http://portal.udea.edu.co/wps/wcm/ connect/udea/c774d2eb-004d-4a31-90d7-a62a0b27a8c6/ proyectos-admitidos-definitivo-csha2016.pdf?MOD= AJPERES\&CVID=IAIRCGg. Acceso en: dic. 22, 2016.

Sugimoto, C.R. et al. Scholarly use of social media and altmetrics: A review of the literature. Journal of the Association for Information Science and Technology, v.68, n.9, p.2037-2062, 2017. Doi: http://dx.doi.org/10.1002/asi.23833

Thelwall, M. et al. Do Altmetrics work? Twitter and ten other social web services. PloS One, v.8, n.5, p.64-84, 2013. Doi: http://dx.doi.org/10.1371/journal.pone.0064841

Torres-Salinas, D. et al. Altmetrics: New Indicators for scientific communication in Web 2.0. Comunicar, v.21, n.41, p.53-60, 2013. Doi: http://dx.doi.org/10.3916/C41-2013-05

Tunger, D. et al. Altmetrics: State of the Art and a Look into the Future. In: Jibu, M.; Osabe, Y. (Ed.). Scientometrics. London: IntechOpen, 2018. p.123-134. Doi: http://dx.doi.org/10.5772/ intechopen.76874

Uribe-Tirado, A. Percepciones, políticas y formación hacia la visibilidad académica y científica entre profesoresinvestigadores de la Universidad de Antioquia. In: Conferencia Internacional BIREDIAL-ISTEC, 15., 2015. Barranquilla: Universidad del Norte, 2015. Disponible en: http://eprints.rclis.org/28499/. Acceso en: Enero 23, 2019.

Uribe-Tirado, A.; Alhuay-Quispe, J. Estudio métrico de ALFIN en Iberoamérica: de la bibliometría a las altmetrics. Revista Española de Documentación Científica, v.40, n.3., p.180, 2017. Doi: http://dx.doi.org/10.3989/redc.2017.3.1414

Vélez-Cuartas, G. et al. Indicadores de vinculación con el entorno para Unidades de Gestión de Investigación Medellin (Colômbia): Universidad de Antioquia, 2017. Universidad de Antioquia - SIU, Estudio Piloto 2004-2016. Disponible en: http://hdl. handle.net/10495/9119. Acceso en: Enero 25, 2019.

Weller, K. Social media and altmetrics: An overview of current alternative approaches to measuring scholarly impact. In: Welpe, I.M. et al. (Ed.). Incentives and performance. Cham: Springer International Publishing, 2015. p.261-276. Doi: http:// dx.doi.org/10.1007/978-3-319-09785-5_16

Williams, A.E. Altmetrics: An overview and evaluation. Online Information Review, v.41, n.3, p.311-317, 2017. Doi: http:// dx.doi.org/10.1108/OIR-10-2016-0294

Zahedi, Z., Costas, R.; Wouters, P. Mendeley readership as a filtering tool to identify highly cited publications. Journal of the Association for Information Science and Technology, v.68, n.10, p.2511-2521, 2017. Doi: http://doi.org/10.1002/asi.23883 